\title{
Inverter Non-Idealities Override by Repetitive Control
}

\author{
Silverio Bolognani \\ University of Padova \\ Department of Electrical Engineering \\ Via Gradenigo 6/A \\ 35131 Padova - Italy
}

\author{
Luca Peretti and Mauro Zigliotto \\ University of Padova \\ Department of Engineering and Management \\ Stradella San Nicola 3 \\ 36100 Vicenza - Italy
}

\begin{abstract}
High performance sensorless AC drives require the exact knowledge of the motor phase voltages in the whole speed range. The use of voltage sensors may be eschewed if the reference voltage signals, generated by the control algorithm, can be used instead. To this aim, an accurate compensation of most of inverter non-idealities is stringent. This paper presents a novel technique for the cancellation of inverter non-idealities, based on repetitive control. The main advantage is that the compensation automatically includes the IGBT parasitic effects during current zero-crossing, whose exact knowledge is one of the major problems in most of the standard dead-time cancellation techniques. Despite its elaborated theoretical background, the method requires few computational resources and it is easy to implement. Mathematical developments, design hints and an extensive batch of successful experimental tests are included in the paper.
\end{abstract}

\section{INTRODUCTION}

It is well known that the dead-times related voltage distortion in inverter-fed electrical drives negatively affects the performances of both open loop control algorithms (like the $\mathrm{V} / \mathrm{Hz}$ control for induction motor drives) in the low speed region, and sensorless AC drives, which often make use of the voltage references instead of the actual measured ones.

The problem of dead-time distortion compensation has been widely discussed in literature, with the purpose of compensating not only the well-known step-like ideal distortion, but also by including some second-order non-linearities. In [1] a PI regulator is used to compensate the differences between the reference and actual voltage measured with a dedicated circuit. Other solutions ([2]-[3]) try to cancel non idealities by means of IGBT and diode models. Assumed a certain accuracy of the models, these approaches can be quite efficient, at the cost of an increasing computational effort and of a rather complicate off-line measurement batch. Some solutions ([4][6]) use observers that relies on motor parameters, along with their related uncertainties.

Instead of using either models or voltage measurements, further works exploit the harmonic distortion in phase currents to cancel the disturbances in reference voltages. In [7] and [8] two on-line algorithms calculate the voltage compensation trying to minimise the voltage distortion. However, the on-line computational effort and the time required for the algorithm convergence have negative influence on the overall dynamic behaviour. In [9], a repetitive control in the angle domain for an Induction Motor (IM) drive is used, since it is claimed that dead-time distortions are fixed in the angle domain. The main disadvantage is that the transformation between the time domain and the angle domain needs a measurement of both rotor and slip speed, so the method does not fit for sensorless drives.

Nevertheless, due to the periodical nature of voltage distortion at steady state, the use of a repetitive control for deadtime compensation is intriguing. The rather specialized subject of internal model controls (to which the repetitive control belongs to) was firstly investigated around the beginning of the 80's. Perhaps boosted by the use of computers in control applications, the ability to store a whole period of the disturbance signal made possible the practical application of these techniques [10]. One of the first examples of repetitive control application was the rejection of periodic disturbances acting on the track-following servo system of optical disk drives [11]. In recent years, repetitive control has been used in electric drive applications, as for example in [12] in which the torque ripple has been profitably reduced.

In this work, the repetitive control is exploited to generate an application-specific look-up table (LUT) that will work at low current levels, where the behaviour of power modules noticeably deviates from the ideal switch. The LUT is merged with conventional step-like compensation for higher current levels, and then used in the whole IM speed range. The proposed method dodges both IGBT models and voltage measurement. Even the accurate current zero-crossing detection, bottleneck of most standard strategies, is less stringent. A further advantage over existing solutions is the low computational requirement, due to the LUT-based approach, suitable for lowcost applications. Several experimental tests have been carried out to prove the effectiveness of the solution, and its generality as well.

\section{THE DEAD-TIME DISTORTION}

The dead-time distortion for each inverter leg can be expressed as:

$$
u_{d i s t, k n}=\frac{t_{d}}{T_{c}} U_{d c} \operatorname{sgn}\left(i_{k}\right)
$$


where $i_{k}$ is the current of the $k$-phase $(k=a, b, c), T_{c}$ is the PWM switching period, $U_{d c}$ is the dc-link voltage, and $t_{d}$ is equal to:

$$
t_{d}=t_{o f f}-t_{o n}-t_{d, s}
$$

where $t_{\text {off }}$ is the IGBT fall time, $t_{\text {on }}$ is the IGBT rise time, and $t_{d, s}$ is the safe interval between the commutation edges of the upper and lower devices in the inverter leg. In a space vector notation, (1) are expressed by [13]:

$$
\boldsymbol{u}_{d i s t}=\boldsymbol{u}_{s}^{*}-\boldsymbol{u}_{s}=-\frac{4}{3} \frac{t_{d}}{T_{c}} U_{d c} \operatorname{sgn}\left(\boldsymbol{i}_{s}\right)
$$

where:

$$
\operatorname{sgn}\left(i_{s}\right)=\frac{\operatorname{sgn}\left(i_{a}\right)+\operatorname{sgn}\left(i_{b}\right) e^{j \frac{2 \pi}{3}}+\operatorname{sgn}\left(i_{c}\right) e^{j \frac{4 \pi}{3}}}{\left|\operatorname{sgn}\left(i_{a}\right)+\operatorname{sgn}\left(i_{b}\right) e^{j \frac{2 \pi}{3}}+\operatorname{sgn}\left(i_{c}\right) e^{j \frac{4 \pi}{3}}\right|}
$$

As can be seen from (3), the distortion created by the deadtime affects both the amplitude and the phase of the actual voltage vector, with respect to its reference. The voltage distortion (1) can be expressed in a synchronous $d q$ rotating frame, locked to the phase $\vartheta_{i}$ of current space vector, as follows:

$$
\begin{aligned}
& u_{d i s t, d}\left(\vartheta_{i}\right)=\frac{4 t_{d}}{\pi T_{c}} U_{d c}\left[1-\sum_{n=1}^{+\infty}(-1)^{n} \frac{2}{36 n^{2}-1} \cos \left(6 n \vartheta_{i}\right)\right] \\
& u_{d i s t, q}\left(\vartheta_{i}\right)=\frac{4 t_{d}}{\pi T_{c}} U_{d c} \sum_{n=1}^{+\infty}(-1)^{n} \frac{12 n}{36 n^{2}-1} \sin \left(6 n \vartheta_{i}\right)
\end{aligned}
$$

Aside from the constant term in $u_{d i s t, d}$, the distortion can be identified as a sum of $6 n$-th harmonic sinusoidal components $(n=1, \ldots,+\infty)$. It is also worth to note that the distortions expressed in (1) create an homopolar component equal to:

$$
u_{h o m}=\frac{t_{d}}{3 T_{c}} U_{d c}\left(\operatorname{sgn}\left(i_{a}\right)+\operatorname{sgn}\left(i_{b}\right)+\operatorname{sgn}\left(i_{c}\right)\right)
$$

\section{A. IGBT parasitic effects}

The IGBT parasitic effects heavily deteriorate the common step-like dead-time compensation. These effects can be managed by a proper look-up table, whose input and output are the phase current amplitude and the voltage correction, respectively. Quite often, this method is accomplished by either offline measurements or complex IGBT models [2]. Conversely, the proposed technique performs the task as a part of its selfcommissioning procedure. The parasitic capacitances affects IGBT commutations in proximity of the zero-crossing of each current phase. Since there are six zero crossings during a $2 \pi$ rotation of a synchronous frame, the related distortion effect is a sum of $6 n$-th harmonics $(n=1, \ldots,+\infty)$, which overlap the harmonics coming from (5).

\section{THE PROPOSED STEADY STATE ON-LINE PROCEDURE}

The algorithm exploits the presence of periodic components of the disturbance, at steady state, caused by voltage distortion in stator currents. The use of the currents, instead of the voltages, let any voltage measurement to be avoided, which is actually one of the main goals. The procedure consists

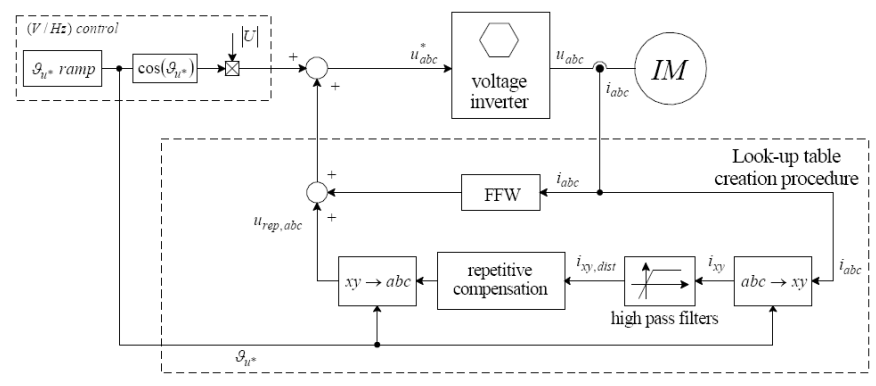

Figure 1. Block scheme of the proposed on-line procedure

of two main steps. The first one, performed on-line at startup and sketched in Figure 1, acquires the phase currents and performs the coordinates transformation into a rotating reference frame fixed to the reference voltage vector. Once the steady-state condition is reached, the procedure is activated. The currents are high-pass filtered to get the $6 n$-th components that, changed in sign, constitute the input to the repetitive block. The compensation output is the sum of the repetitive control output, tuned on the $6 n$-th harmonics in the rotating reference frame (details will be given in Section IV), and a feedforward action for the constant component of (5) that, not being a multiple of the $6 n$-th harmonic, is not included in the repetitive control action (FFW block, Figure 1). The procedure outlined above runs until the current harmonics have been reduced within a specified upper bound. Then, the generated compensation voltage patterns are back-transformed in the $a b c$ reference frame and stored in application-specific look-up tables (LUTs).

The second step, performed during normal drive operations, exploits the prepackaged look-up tables obtained in the first step, which are accessed using the phase currents as input. As said, they return the correction to the voltage reference vector (Figure 1). Since the actual DC-link voltage $U_{d c}$ may differ from the one present during the LUTs creation, the output is corrected by the ratio of the former to the latter.

The compensation is clearly focused on harmonics cancellation, and it inherently includes the IGBT parasitic effects during current zero-crossing, which is one of the major problems in most of the standard techniques. Since the prepackaged look-up tables are created in a specific operating condition, there is an implicit acceptance of sub-optimal cancellation of IGBTs parasitic effects, only for the part that is speed dependant. Nevertheless, experimental results reported in Section $\mathrm{V}$ show that it is quite negligible.

The repetitive control is performed in a $x y$ rotating frame, synchronised with the reference voltage vector phase angle $\vartheta_{u^{*}}$. It is because during the first step the motor is operated by a standard $\mathrm{V} / \mathrm{Hz}$ open-loop control (Figure 1), and then $\vartheta_{u^{*}}$ is a handy, built-in angle. This simple choice makes the proposed compensation suitable for low-cost drives. As well, it can be also regarded as the first step in advanced parameter identification techniques for sensorless drives.

Some concern may arouse because the selected reference frame is fixed to the voltage reference, while the distortion 


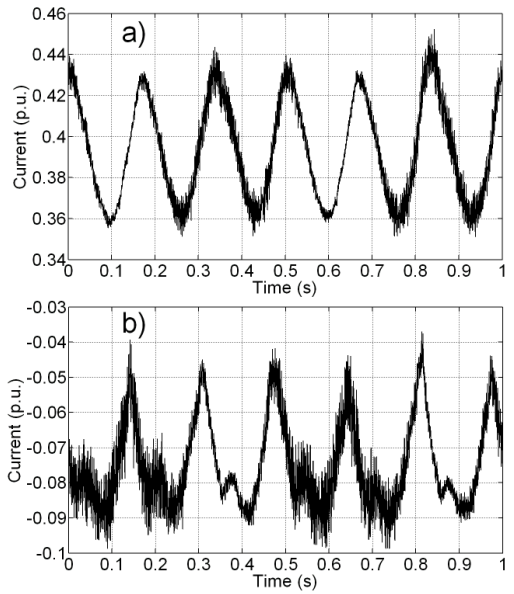

Figure 2. Experimental profiles of stator current - a) $i_{x}$, b) $i_{y}$.

voltages (5) are expressed as function of current angle $\vartheta_{i}$. As a matter of fact, under the hypothesis of a temporarily constant load torque (just for the completion of the first on-line step), in steady-state condition $\vartheta_{u^{*}}$ exhibits a constant phase advance with respect to $\vartheta_{i}$. Therefore, the $6 n$-th harmonics distortion on the stator currents components $i_{s x}$ and $i_{s y}$ remains clearly recognisable. An example is reported in Figure 2, which reports $i_{x}$ and $i_{y}$ with the reference voltage vector rotating at a frequency of $1 \mathrm{~Hz}$.

\section{THE REPETITIVE CONTROL-BASED COMPENSATION}

In this paper, the repetitive control technique is applied to cancel the periodic disturbances due to dead-times at steady state in a inverter-fed motor drive. Mathematically, the repetitive block of Figure 1 can be described by the following transfer function in the $z$-domain [10]:

$$
R E P(z)=K_{R E P} \frac{z^{K_{a}}}{z^{M}-F_{R}(z)}
$$

where $F_{R}(z)=\frac{N_{F R}(z)}{D_{F R}(z)}$ is a filter that limits the repetitive control gain at high frequency [12]. The parameter $K_{a}$ introduces a leading action on the controller to compensate the delay of the process (in this case, the cascade of the voltage inverter and IM), while $K_{r e p}$ is the controller gain. In the case of $F_{R}(z)=1$, the repetitive block shows an infinite gain for the frequencies multiple of $1 /\left(M T_{c}\right)$, and $M$ can be selected to match the repetitive control resonant frequency with that of the input disturbance. In presence of a filter $F_{R}(z)$, the parameter $M$ is appropriately modified to take into account the phase delay introduced by $F_{R}(z)$ itself. In the present case of a linear-phase FIR filter, it is sufficient to subtract from $M$ the filter group delay, which is constant. Obviously, since $M$ is an integer, the filter taps have to be designed so to let the group delay be a multiple of the sampling frequency.

It is well known that dead times effects are better highlighted at low voltages. To this purpose, it has been found that for a wide variety of motors a standard open loop $\mathrm{V} / \mathrm{Hz}$ control with a voltage reference vector rotating at $16.67 \mathrm{~Hz}$

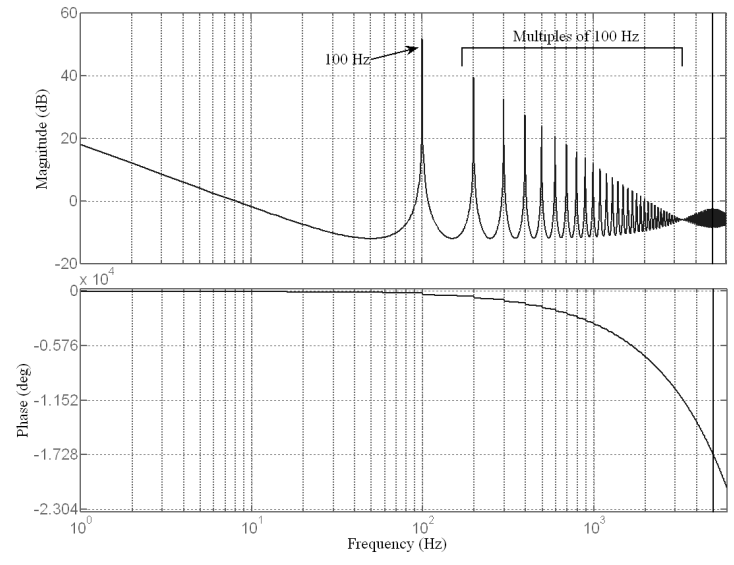

Figure 3. Bode diagram of the repetitive block with $F_{R}(z)$ filter

(for an IM with one pole pair, it corresponds to $1000 \mathrm{rpm}$ ) is a suitable choice. As a consequence, the distortion-related 6-th harmonic is located at $100 \mathrm{~Hz}$, which also becomes the resonant frequency of the repetitive control. A sample frequency $F_{c}=10 \mathrm{kHz}$ (control period $T_{c}=100 \mu \mathrm{s}$ ) leads to the setting $M=100$.

The filter $F_{R}(z)$ is a simple moving average filter with three taps. Its corresponding group delay is $T_{c}$, perfectly compensated by choosing $M=99$. The Bode diagram of the repetitive block of Figure 1, comprehensive of the $F_{R}(z)$ filter, is shown in Figure 3.

\section{A. Current FIR filters}

Although not strictly necessary, the $a b c$ phase currents are filtered with another moving average FIR filter with three taps, to get smoother look-up tables, and only for the time needed to create the tables. To avoid that the filter phase delay affects the look-up tables, a simple compensation technique is used. Let a vector $\boldsymbol{g}_{f}=g_{f, x}+j g_{f, y}$, rotating at a constant speed equal to $\omega$, be the output $\boldsymbol{g}_{f}$ of a filter, delayed by $\vartheta$ with respect to the filter input. A new vector $\boldsymbol{g}$, with a phase advance of $\vartheta$ with respect to $\boldsymbol{g}_{f}$ is sought. Mathematically, one can write:

$$
\boldsymbol{g}_{f}=\boldsymbol{g} e^{-j \vartheta}=g e^{j(\omega t-\vartheta)}=g[\cos (\omega t-\vartheta)+j \sin (\omega t-\vartheta)]
$$

If $\vartheta$ is small with respect to the rotating period of $\boldsymbol{g}$, the following approximations can be made:

$$
\begin{aligned}
& \cos (\omega t-\vartheta) \approx \cos (\omega t)+\vartheta \sin (\omega t) \\
& \sin (\omega t-\vartheta) \approx \sin (\omega t)-\vartheta \cos (\omega t)
\end{aligned}
$$

and (8) becomes:

$$
\begin{aligned}
\boldsymbol{g}_{f} & =g[\cos (\omega t)+j \sin (\omega t)]+g \vartheta[\sin (\omega t)-j \cos (\omega t)] \\
& =\boldsymbol{g}(1-j \vartheta)
\end{aligned}
$$

that yields

$$
\boldsymbol{g}=\frac{\boldsymbol{g}_{f}}{1-j \vartheta}=\frac{1+j \vartheta}{1+\vartheta^{2}} \boldsymbol{g}_{f}
$$

The denominator of (10) can be expressed by a Taylor expansion:

$$
\boldsymbol{g} \approx(1+j \vartheta)\left(1-\vartheta^{2}\right) \boldsymbol{g}_{f}
$$


whose $x y$ components are

$$
\begin{aligned}
& g_{x}=\left(1-\vartheta^{2}\right)\left(g_{f, x}-\vartheta g_{f, y}\right) \\
& g_{y}=\left(1-\vartheta^{2}\right)\left(g_{f, x}+\vartheta g_{f, y}\right)
\end{aligned}
$$

Since $\vartheta$ is the phase delay of a FIR filter, which is inherently linear, the (12) are of immediate application.

\section{B. Stability analysis}

The repetitive control is activated at steady state, in a precise and predetermined working condition, which in the present work was characterised by a reference voltage vector frequency of $16.67 \mathrm{~Hz}$, at no load. The following stability analysis has anyway general validity.

The IM state-space model, expressed in a generic frame rotating at $\omega_{g}$, is given by

$$
\begin{aligned}
\frac{d}{d t}\left[\begin{array}{c}
i_{s} \\
\lambda_{r}
\end{array}\right] & =\left[\begin{array}{ll}
A_{11} & A_{12} \\
A_{21} & A_{22}
\end{array}\right]\left[\begin{array}{c}
i_{s} \\
\lambda_{r}
\end{array}\right]+\left[\begin{array}{c}
B_{1} \\
0
\end{array}\right] u_{s} \\
y & =\left[\begin{array}{ll}
1 & 0
\end{array}\right]\left[\begin{array}{l}
i_{s} \\
\lambda_{r}
\end{array}\right]
\end{aligned}
$$

where vectors and matrices assume the following form:

$$
\begin{aligned}
& i_{s}=\left[\begin{array}{ll}
i_{s x} & i_{s y}
\end{array}\right]^{T} \quad \text { stator currents } \\
& \lambda_{r}=\left[\begin{array}{ll}
\lambda_{r x} & \lambda_{r y}
\end{array}\right]^{T} \quad \text { rotor fluxes } \\
& u_{s}=\left[\begin{array}{ll}
u_{s x} & u_{s y}
\end{array}\right]^{T} \quad \text { stator voltages } \\
& A_{11}=-\frac{1}{L_{t}}\left(R_{s}+R_{r} \frac{L_{m}^{2}}{L_{r}^{2}}\right) I-\omega_{g} J \\
& A_{12}=\frac{R_{r}}{L_{t}} \frac{L_{m}}{L_{r}^{2}} I-\omega_{m e} \frac{1}{L_{t}} \frac{L_{m}}{L_{r}} J \\
& A_{21}=\frac{R_{r} L_{m}}{L_{r}} I \\
& A_{22}=-\frac{R_{r}}{L_{r}} I-\left(\omega_{g}-\omega_{m e}\right) J \\
& B_{1}=\frac{1}{L_{t}}, \\
& I=\left[\begin{array}{ll}
1 & 0 \\
0 & 1
\end{array}\right] \text {, } \\
& J=\left[\begin{array}{cc}
0 & -1 \\
1 & 0
\end{array}\right]
\end{aligned}
$$

$R_{s}, R_{r}, L_{s}, L_{r}$ are the stator and rotor resistances and selfinductances respectively, $L_{m}$ and $L_{t}$ are the magnetising and transient inductances and $\omega_{m e}$ is the electromechanical motor speed. In this work, $\omega_{g}$ is set equal to the reference voltage vector speed, so to obtain the IM model in the $x y$ reference frame. It is worth to note that in the $x y$ frame there is an axes cross-coupling that cannot be neglected a-priori. From (13) and its specifications, the transfer functions between the inputs $u_{x}, u_{y}$ and the outputs $i_{x}, i_{y}$ are readily obtained. Consequently, the block scheme for the stability analysis can be rearranged as depicted in Figure 4.

For each $x y$ axis, the control input is the difference between the actual current ripple and its reference, which is obviously null (Figure 4). At a glance, the $y$ axis can be viewed from

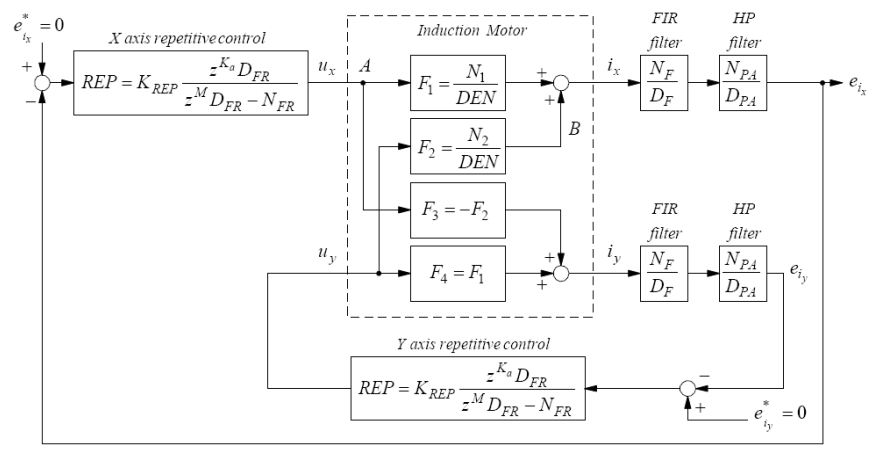

Figure 4. Block scheme for the stability analysis of the repetitive control

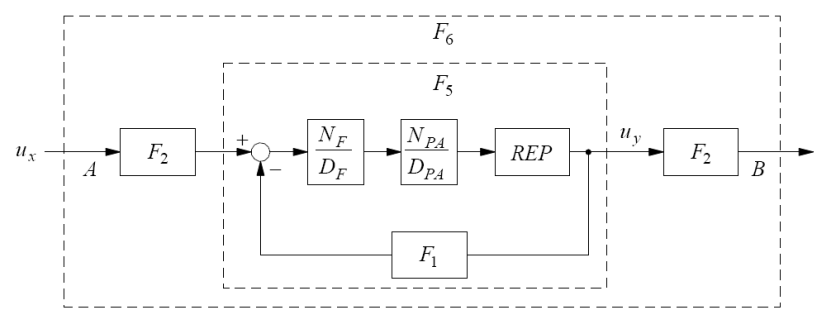

Figure 5. Subsystem for the stability analysis of the y axis loop

point $A$ to point $B$ as a separate subsystem, described by the block scheme of Figure 5 , in which it is

$$
\begin{aligned}
& F_{5}=\frac{K_{R E P} \frac{N_{F}}{D_{F}} \frac{N_{P A}}{D_{P A}} z^{K_{a}}}{z^{M}-[\underbrace{\frac{N_{F R}}{D_{F R}}-K_{R E P} \frac{N_{F}}{D_{F}} \frac{N_{P A}}{D_{P A}} \frac{N_{1}}{D E N} z^{K_{a}}}_{H_{2}(z)}]} \\
& F_{6}=F_{2}^{2} F_{5}
\end{aligned}
$$

As reported in [12], a sufficient condition for the $y$ axis stability is:

$$
\left|H_{2}(z)\right|_{z=e^{j \omega T_{c}}}<1
$$

in which $\omega$ sweeps from zero to the Nyquist frequency $F_{N}=F_{c} / 2$. Figure 6 shows the $H_{2}(z)$ plot in the Nyquist coordinates at no-load, for $\omega \in\left[0, F_{N}\right]$. It has been obtained with the parameters of the IM 1 (see Appendix) and by setting $K_{\text {rep }}=0.03$ and $K_{a}=3$. The condition (15) is satisfied and thus $F_{5}$ is stable. Other simulations have been performed by varying $K_{r e p}$ from 0.1 to 10 times the nominal values, and by varying the slip speed from 0 to $5 \%$ of $\omega_{g}$. It has been experienced that $H_{2}(z)$ is scarcely affected by such variations, and it always remains within the unity circle.

Since $F_{2}$ is stable, from (14) the stability of $F_{5}$ implies that of $F_{6}$, too. With reference to the block scheme of Figure 7, the same procedure can now be applied to the stability analysis of the $x$ axis. The transfer function $e_{i_{x}} / e_{i_{x}^{*}}$ is calculated by 


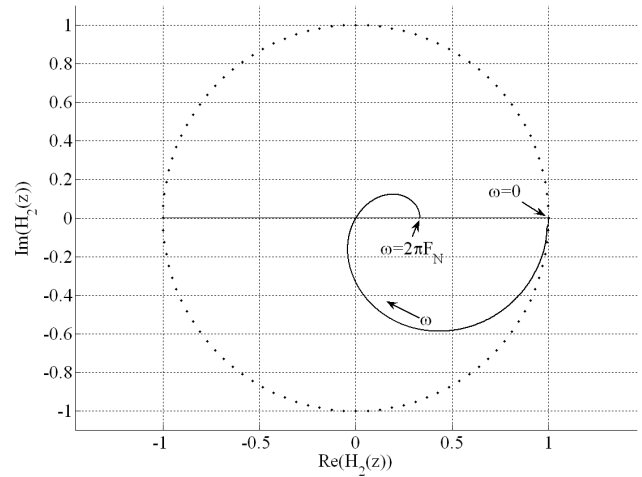

Figure 6. $H_{2}(z)$ as function of $z=e^{j \omega T_{c}}$

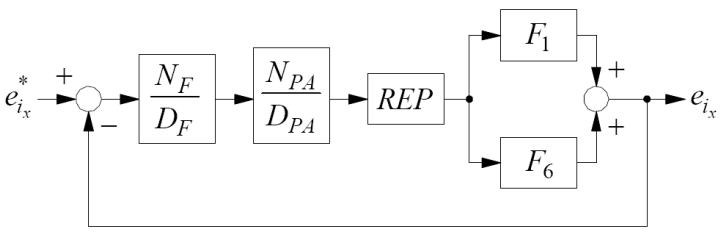

Figure 7. Block scheme for the stability analysis of the $\mathrm{x}$ axis loop

expanding the $R E P$ block, obtaining

$$
\frac{e_{i_{x}}}{e_{i_{x}^{*}}}=\frac{K_{R E P} \frac{N_{F}}{D_{F}} \frac{N_{P A}}{D_{P A}}\left(F_{1}+F_{6}\right) z^{K_{a}}}{z^{M}-[\underbrace{\frac{N_{F R}}{D_{F R}}-K_{R E P} \frac{N_{F}}{D_{F}} \frac{N_{P A}}{D_{P A}}\left(F_{1}+F_{6}\right) z^{K_{a}}}_{H_{1}(z)}]}
$$

As before, the system is stable if:

$$
\left|H_{1}(z)\right|_{z=e^{j \omega T_{c}}}<1
$$

with $\omega$ ranging from zero to $F_{N}$. Using the same parameters as for the $H_{2}(z)$ analysis, and by performing the same robustness analysis, it has been found that the diagram of $H_{1}(z)$ in the Nyquist coordinates always resides within the unity circle, proving the overall stability of the proposed repetitive control.

\section{Look-up table creation and elaboration}

An output example of the self-commissioning procedure is reported in Figure 8, which shows the look-up table for the $a$ phase of IM 1.

The profile of Figure 8 is directly obtained by adding the repetitive control output and the FFW contribute (Figure 1), but it still needs some further post-processing to be effective. As depicted in Figure 8, the multiple step-like behaviour is due to to the lack of information about the homopolar component (6), which is lost in the $x y$ reference frame. For the sake of simplicity, the homopolar component can be recovered by multiplying the values in Figure 8 by the term 3/2, taking care of eliminating the extremes of the look-up table. These outer side values are not strictly necessary, since they correspond to higher phase currents, for which a conventional compensation

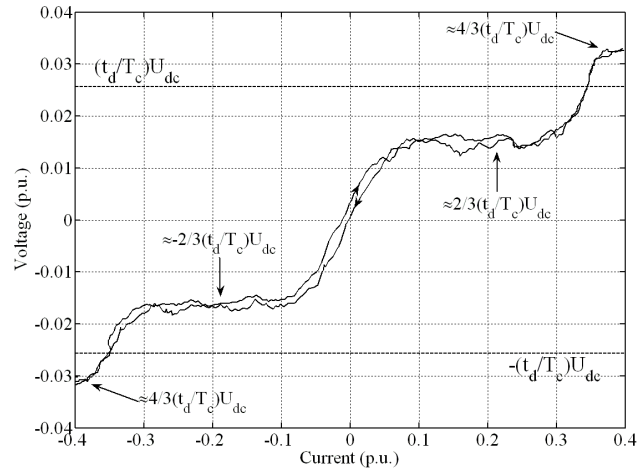

Figure 8. Experimental acquisition of a look-up table prior to processing actions

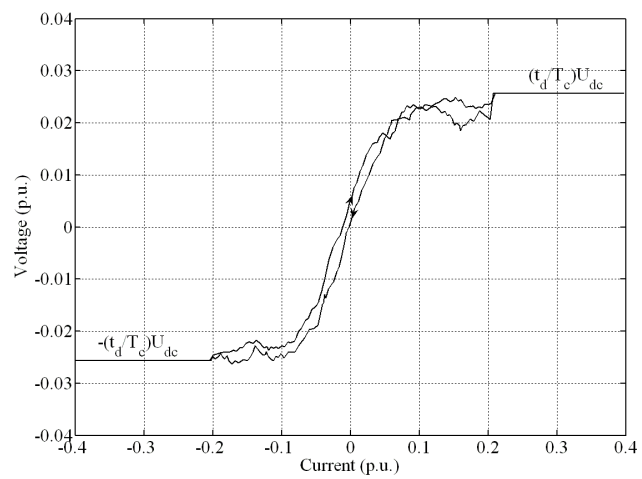

Figure 9. Experimental acquisition of a look-up table after processing actions

Table I

MOTOR PARAMETERS USED IN THE EXPERIMENTAL PHASE

\begin{tabular}{c|c|c|c}
\hline & IM 1 & IM 2 & IM 3 \\
\hline \hline Nominal power & $1.2 \mathrm{~kW}$ & $0.75 \mathrm{~kW}$ & $3 \mathrm{~kW}$ \\
\hline Nominal voltage & $400 \mathrm{~V}$ & $200 \mathrm{~V}$ & $400 \mathrm{~V}$ \\
\hline Nominal current & $2.8 \mathrm{~A}$ & $2.5 \mathrm{~A}$ & $8 \mathrm{~A}$ \\
\hline Nominal speed & $3000 \mathrm{rpm}$ & $4000 \mathrm{rpm}$ & $1500 \mathrm{rpm}$ \\
\hline
\end{tabular}

expressed by (1) can be used instead. The resulting postprocessed $a$ phase current LUT is shown in Figure (9).

As expected, for low current levels the ideal step of (1) is smoothed by IGBT and diode parasitic effects. Moreover, and according to the common understanding of the commutation phenomena, the small hysteresis shows that there is a slightly different behaviour for negative and positive derivative of the phase currents. Consequently, a precise compensation could make use two different look-up tables for the two cases.

\section{EXPERIMENTAL RESULTS}

The proposed procedure has been evaluated on three different induction motors, whose parameters are reported in Table I, making use of a fast control prototyping laboratory setup. The filter and repetitive block parameters are the same as those used in the stability analysis of Section IV-B for all IMs, except for $K_{r e p}$ that has been experimentally increased from 0.03 to 0.5 in the specific operating point, reducing 

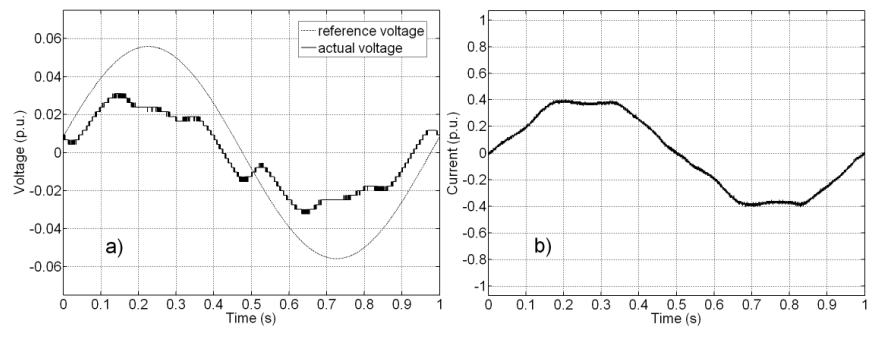

Figure 10. a) phase voltage and b) current: no compensation, $60 \mathrm{rpm}$
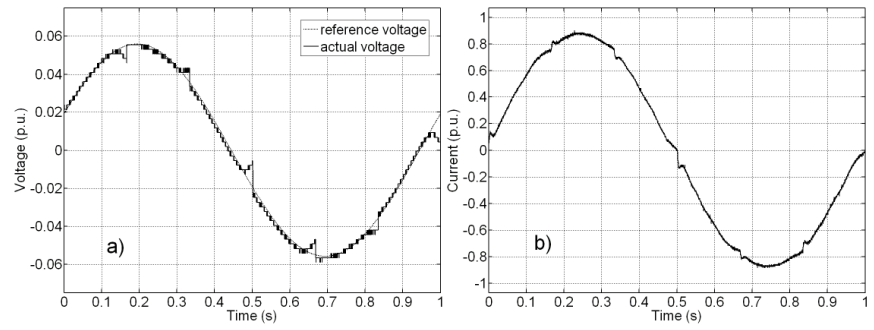

Figure 11. a) phase voltage and b) current: step-like compensation, $60 \mathrm{rpm}$
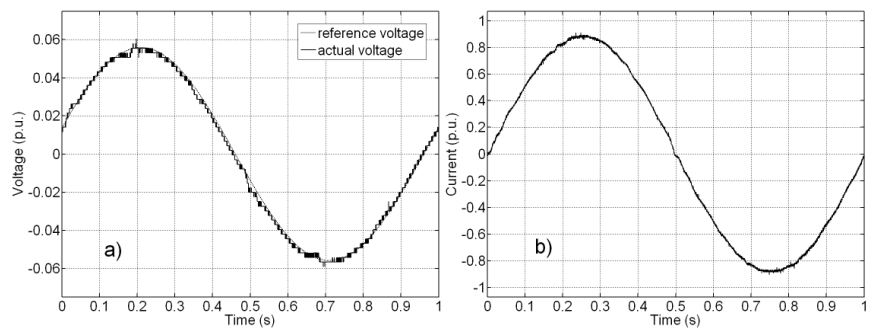

Figure 12. a) phase voltage and b) current: proposed compensation, $60 \mathrm{rpm}$

Table II

COMPARISON OF VOLTAGE THD (EXPERIMENTAL RESULTS, @60 RPM)

\begin{tabular}{c|c|c|c}
\hline & $\begin{array}{c}\text { THD } \\
\text { no compensation }\end{array}$ & $\begin{array}{c}\text { THD } \\
\text { conventional }\end{array}$ & $\begin{array}{c}\text { THD } \\
\text { proposed }\end{array}$ \\
\hline IM 1 & 0.23 & 0.17 & 0.09 \\
\hline IM 2 & 0.30 & 0.21 & 0.05 \\
\hline IM 3 & 0.37 & 0.17 & 0.05 \\
\hline
\end{tabular}

execution time while preserving the system stability. Figure 10,11 and 12 report a comparison between voltage and current measurements for IM 1 at no load with a rotating frequency of $1 \mathrm{~Hz}(60 \mathrm{rpm})$, in case of no dead-time compensation, conventional step-like compensation and compensation based on LUT of Figure 9 respectively. Very similar results are obtained with all the other IMs. As a measure of the effectiveness of the proposed solution, Table II e III collect the voltage and current THD obtained from experimental measurements on the different prototypes. The advantages of the proposed solution are clear.

\section{CONClusions}

In this paper, a procedure for an accurate compensation of inverter non-idealities have been presented. The proposed approach uses repetitive control to create application-specific look-up tables, which can be used instead of a conventional
Table III

COMPARISON OF CURRENT THD (EXPERIMENTAL RESULTS, @60 RPM)

\begin{tabular}{c|c|c|c}
\hline & $\begin{array}{c}\text { THD } \\
\text { no compensation }\end{array}$ & $\begin{array}{c}\text { THD } \\
\text { conventional }\end{array}$ & $\begin{array}{c}\text { THD } \\
\text { proposed }\end{array}$ \\
\hline IM 1 & 0.09 & 0.08 & 0.05 \\
\hline IM 2 & 0.11 & 0.05 & 0.02 \\
\hline IM 3 & 0.12 & 0.09 & 0.03 \\
\hline
\end{tabular}

step-like compensation in an IM electric drive, while maintaining a low-cost profile and low computational effort.

A complete stability analysis has been developed, and extensive experimental results on three IMs have been presented. The comparison between the voltage and current THD in the three cases further confirms the effectiveness of the proposed solution, which is going to be implemented by an industrial partner.

\section{APPENDIX \\ IM 1 ELECTRICAL PARAMETERS}

\begin{tabular}{c|c|c|c}
\hline$R_{s}$ & $2.74 \Omega$ & $R_{r}$ & $1.70 \Omega$ \\
\hline$L_{s}$ & $0.161 \mathrm{H}$ & $L_{r}$ & $0.161 \mathrm{H}$ \\
\hline$L_{m}$ & $0.157 \mathrm{H}$ & $p$ & 1 \\
\hline
\end{tabular}

\section{REFERENCES}

[1] J. Jung, K. Nam, A PI-Type Dead-Time Compensation Method for Vector-Controlled GTO Inverters, IEEE Transactions on Industry Applications, Vol. 34, No. 3, May/June 1998.

[2] A. R. Munoz, T. A. Lipo, On-Line Dead-Time Compensation Technique for Open-Loop PWM-VSI Drives, IEEE Transactions On Power Electronics, Vol. 14, No. 4, pp. 683-689, July 1999.

[3] J. Holtz, J. Quan, Sensorless Vector Control of Induction Motors at Very Low Speed Using a Nonlinear Inverter Model and Parameter Identification, IEEE Transactions On Industry Applications, Vol. 38, No. 4, July/August 2002.

[4] C. Attaianese, G. Tomasso, Predictive Compensation of Dead-Time Effects in VSI Feeding Induction Motors, IEEE Transactions on Industry Applications, Vol. 37, No. 3, May/June 2001.

[5] H.-S. Kim, H.-T. Moon and M.-J. Youn, On-Line Dead-Time Compensation Method Using Disturbance Observer, IEEE Transactions On Power Electronics, Vol. 18, No. 6, November 2003.

[6] N. Urasaki, T. Senjyu, K. Uezato and T. Funabashi, An Adaptive Dead-Time Compensation Strategy for Voltage Source Inverter Fed Motor Drives, IEEE Transactions on Power Electronics, Vol. 20, No. 5, September 2005.

[7] H. Zhao, Q. M. J. Wu and A. Kawamura, An Accurate Approach of Nonlinearity Compensation for VSI Inverter Output Voltage, IEEE Transactions on Power Electronics, Vol. 19, No. 4, July 2004.

[8] A. Cichowski, J. Nieznanski, -Tuning Dead-Time Compensation Method for Voltage-Source Inverters, IEEE Power Electronic Letters, Vol. 31, No. 2, June 2005.

[9] L. Ben-Brahim, On the Compensation of Dead Time and Zero-Current Crossing for a PWM-Inverter-Controlled AC Servo Drive, IEEE Transactions On Industrial Electronics, Vol. 51, No. 5, October 2004.

[10] G. Hillerström and K.Walgama, Repetitive Control Theory and Applications - A Survey, Proc. of the 13th IFAC World Congress, Vol. D, pp.1-6, San Francisco, July 1996.

[11] J. H. Moon, M. N. Lee, M. J. Chung, Repetitive Control for the TrackFollowing Servo System of an Optical Disk Drive, IEEE Trans. on Control Systems Technology, pp. 663-670, Sept.1998.

[12] P. Mattavelli, L. Tubiana, M. Zigliotto, Torque-Ripple Reduction in PM Synchronous Motor Drives Using Repetitive Current Control, IEEE Transactions on Power Electronics, Vol. 20, No. 6, November 2005

[13] S.Bolognani, M. Zigliotto, Self-Commissioning Compensation of Inverter Non-Idealities for Sensorless AC Drives Applications, Proceedings of IEE International Conference on Power Electronics Machines and Drives, PEMD'02, pp.30-37, Bath, UK, 2002. 